Relations industrielles

Industrial Relations

\title{
Politique et technique de direction du personnel, par Jean Diverrez (4e édition), Paris, Entreprise Moderne d'Édition, 1970, 291 pp.
}

\section{André Roy}

Volume 28, numéro 1, 1973

URI : https://id.erudit.org/iderudit/028383ar

DOI : https://doi.org/10.7202/028383ar

Aller au sommaire du numéro

Éditeur(s)

Département des relations industrielles de l'Université Laval

ISSN

0034-379X (imprimé)

1703-8138 (numérique)

Découvrir la revue

Citer ce compte rendu

Roy, A. (1973). Compte rendu de [Politique et technique de direction du personnel, par Jean Diverrez (4e édition), Paris, Entreprise Moderne d'Édition, 1970, 291 pp.] Relations industrielles / Industrial Relations, 28(1), 221-222. https://doi.org/10.7202/028383ar

Tous droits réservés @ C Département des relations industrielles de l'Université Laval, 1973
Ce document est protégé par la loi sur le droit d'auteur. L’utilisation des services d'Érudit (y compris la reproduction) est assujettie à sa politique d'utilisation que vous pouvez consulter en ligne.

https://apropos.erudit.org/fr/usagers/politique-dutilisation/ 
sur la fausse hypothèse (des méthodes de corrélation et de coefficients techniques fixes) que les relations observées fournissent un guide valable à ceux qui prennent des décisions en matière d'éducation et de main-d'oeuvre.

L'examen des modèles présentés et des limites suggérées caractérisant l'effort de planification de l'éducation et de la main-d'oeuvre nous amène à soulever la question à savoir si, à toute fin pratique, la planification en ces domaines n'est pas un exercice sophistiqué de futilité inutile? Les limites en sont si grandes qu'on se demande si une telle planification peut réellement diriger la prise de décision sur le marché du travail. Souvent, si les projections et prévisions sont exactes, on s'aperçoit rapidement que c'est peut-être beaucoup plus le fruit d'une coïncidence que d'une démarche scientifique (ex. : Swerldoff, Monthly Labor Review, nov. 1969).

De toute façon, cet ouvrage est excellent sur plusieurs points. Les données présentées, les nombreuses limites soulevées, et les problèmes méthodologiques expliqués devraient faire comprendre au nouvel initié et rappeler au planificateur et à l'usager de résultats la vraie nature de la planification des ressources humaines. Il y a évidemment encore énormément de chemin à parcourir en ce domaine. J'ai l'impression que la réponse aux nombreux problèmes de planification ne se retrouvera pas dans la mathématique mais plutôt dans une compréhension plus grande du marché du travail et du comportement des personnes.

\section{Jean SEXTON}

\section{Politique et technique de direction du} personnel, par Jean Diverrez (4e édition), Paris, Entreprise Moderne d'Edition, 1970, $291 \mathrm{pp}$.

La direction du personnel est une discipline relativement nouvelle. C'est pourquoi il est assez rare de pouvoir tomber sur un ouvrage qui n'étant pas trop spécialisé est de nature à apporter aux praticiens de la profession ainsi qu'aux chefs d'entreprise et aux cadres un instrument de travail utile même s'il a été écrit d'abord pour le public français.
L'ouvrage se divise en trois parties distinctes : la direction du personnel, les techniques de gestion du personnel et la psychosociologie de l'entreprise. Il vise, dans son ensemble, à réunir les conditions essentielles à une bonne gestion de même qu'à protéger les intérêts matériels et moraux, individuels et collectifs, de l'employeur et des salariés.

Dans la première partie, l'auteur étudie successivement la politique, l'administration, la fonction et le service du personnel, décrivant d'une façon très claire en particulier les fonctions qui devraient se trouver à l'intérieur d'un bon service du personnel ou s'y rattacher comme celles du médecin du travail, de l'assistante sociale, de la conseillère du travail, de l'ingénieur, de l'agent de sécurité, du psychotechnicien, de l'animateur de formation.

La deuxième partie est consacrée aux techniques de gestion proprement dites : choix et méthodes de sélection, organisation du service d'embauche et son fonctionnement, formation et perfectionnement du personnel et des agents de maîtrise, appréciation, mutation et promotion des employés, rémunération et définition des fonctions.

Enfin, les derniers chapitres portent sur la psychosociologie de l'entreprise, c'est-à-dire sur l'aménagement de relations humaines valables dans le milieu de travail, l'exercice de la discipline et, finalement, sur quelques aspects particuliers des relations de travail, en particulier sur la femme au travail et sur la situation des jeunes ouvriers.

Dans le dernier chapitre, dont on pourrait dire qu'il est la conclusion de l'ouvrage, l'auteur, considérant l'évolution psychologique des travailleurs, aborde brièvement la question des besoins fondamentaux et des aspirations profondes des travailleurs : besoin de réalisation et d'expression de soi, besoin de protéger et d'être protégé, besoin de croître, de s'affirmer, de se donner, d'avoir une véritable vie sociale. On peut en particulier retenir ce conseil de l'auteur :

«Pour diriger efficacement le personnel il est nécessaire de connaître la nature profonde des hommes. Ignorer les forces qui les poussent à agir et ne pas en tenir compte aura pour consé- 
quence de faire naître des sentiments de frustration. Ceux-ci, nous enseignent la psychologie, se traduisent obligatoirement par l'un des trois comportements suivants : l'agressivité, la fuite ou la compensation; agressivité vis-à-vis de la direction, des cadres et de la mâ̂trise, fuite devant le travail, les responsabilités, et disparition de la conscience professionnelle ; compensation, par la recherche d'autres centres d'intérêt que le travail, le jeu ou l'alcool, par exemple, et instabilité du travailleur qui devient l'un de ces éternels insatisfaits s'imaginant qu'un changement d'emploi leur apportera une amélioration».

On se rend donc compte que l'auteur, sans négliger le côté strictement technique de la gestion du personnel, attache une importance de bon aloi à leur aspect humain.

L'ouvrage fournit, à la fin de chaque chapitre une bibliographie intéressante qui permet de voir en même temps qu'il s'agit d'une oeuvre assez dense tout de même que l'auteur a voulu écrire dans une langue décantée de cette sorte de vocabulaire technique qui rend parfois ces sortes de travaux quasi inaccessibles aux profanes. On pourrait ajouter, comme il s'agit d'un ouvrage publié en France et qui reste assez schématique, qu'on $\mathrm{y}$ relève un nombre considérable de termes bien français dont la connaissance pourrait être fort utile à ceux qui chez nous, devant souvent recourir à des manuels rédigés en langue anglaise, sont souvent dans l'obligation d'improviser la traduction à pied levé.

\section{André ROY}

\section{The History of Management Thought,} par Claude George, Jr., Second Edition, Englewood Cliffs, N.J., PrenticeHall, Inc. 1972, 223 pp.

Exposer en deux cents pages l'histoire du management depuis les civilisations les plus reculées en passant par le moyen-âge, la révolution industrielle et les développements des méthodes scientifiques jusqu'à nos jours est un défi extraordinaire.

L'auteur a su éviter les écueils d'une telle entreprise dont les non moindres étaient sûrement une simplification sommaire et le romanesque. Sans doute, dans un sujet aussi vaste, il a été amené à faire des choix. Mais il a retenu l'essentiel et le présente d'une façon synthétique claire, ordonnée et logique. Le tout est complété par une excellente bibliographie choisie.

Cet ouvrage peut être un instrument utile tant aux professeurs et aux. élèves qu'aux praticiens dans le domaine de la gestion.

\section{Gérard DION}

\section{Bargaining : Monopoly versus Union}

Power, by George de Menil, The Joint Center for Urban Studies of The Massachusetts Institute of Technology and Harvard University. The MIT Press, Cambridge, Massachusetts and London, England, 1971, 123 pp.

This book tackles the difficult subject of wage determination in the labor markets of highly unionized and concentrated industries where the standard models of competition, monopoly, and monopolistic competition do not apply. It attempts to bridge the gap between untested, abstract bargaining models and empirical studies that relate wages to 《bargaining variables 》 without the benefit of formal theory. To do this the study derives a wage equation from a bargaining model and then tests this equation on data for manufacturing industries in the United States, drawing conclusions that have important: implications for income distribution and for the analysis of union - non-union wage differentials.

The study presents a survey of some bargaining theories, selects one that the author finds most applicable -- Nash' theory of bargaining which is a branch of the theory of games of Von Neumann and Morgenstern - and from it constructs a model of the firm under bilateral monopoly. Assumptions are made concerning the product demand curve, production function, capital supply, supply of union members and the utility functions of the employer and the union. These assumptions plus two hypotheses from Nash's theory determine the wage rate, employment, capital stock, output, price and profits under bilateral monopoly.

The bargaining wage equation derived from the Nash bilateral monopoly mo- 